\title{
El quehacer pedagógico del docente de Educación física desde su imaginario
}

\author{
Nicolás Guarnizo Carballo
}

\section{Universidad de los Llanos (Colombia) \\ Email: nicolasgreeu@gmail.com}

RESUMEN: Este artículo de investigación hace referencia al imaginario simbólico que tiene el maestro sobre la dimensión lúdica en su quehacer pedagógico. Es por eso que el objetivo general de investigación fue determinar cuál era la imaginación simbólica que tienen los docentes de la ciudad de Villavicencio (Colombia). De este modo, desde una perspectiva fenomenológica, se abordó el significado de esta dimensión que se encuentra dentro de los ambientes educativos y el imaginario simbólico de los docentes. El enfoque utilizado fue el mixto, con un método descriptivo. En las técnicas de recolección de información se manejó la encuesta, la observación y la revisión documental, para la triangulación de la información. Se determinó que el imaginario simbólico del maestro de educación física se encuentra direccionado en los deportes convencionales y en la no planeación de las clases, por este motivo, se aleja de otras manifestaciones lúdicas que posteriormente afectaría el proceso de enseñanza y aprendizaje. De acuerdo con lo anterior, la orientación educativa no se encuentra articulada con las competencias que debe tener el docente, por lo tanto, los objetivos de aprendizaje se deterioran en los espacios de formación estudiantil.

PALABRAS CLAVE: Docente, Educación, Estrategias, Símbolo, Pedagogía

\section{The pedagogical task of the teacher of Physical Education from its symbolic imaginary}

ABSTRACT: This research article refers to the symbolic imaginary that the teacher has about the playful dimension in his pedagogical work. That is why the general objective of research was to determine what the symbolic imagination of the teachers of the city of Villavicencio (Colombia). In this way, from a phenomenological perspective, the meaning of this dimension that is within the educational environments was addressed and the symbolic imaginary of teachers. The approach used was the mixed one, with a descriptive method. In the information collection techniques, the survey, observation and documentary review were handled, thus the triangulation of the information was carried out. It was determined that the symbolic imaginary of the physical education teacher is addressed in conventional sports and in the non-planning of classes, for this reason, it moves away from other 
recreational manifestations that would subsequently affect the teaching and learning process. According to the above, the educational orientation is not articulated with the competencies that the teacher must have, therefore, the learning objectives deteriorate in the spaces of student training.

KEY WORDS: Teacher, Education, Strategies, Symbol, Pedagogy.

\section{INTRODUCCIÓN}

El docente en Educación Física tiene que manejar un discurso pedagógico sobre la dimensión lúdica y más allá de ese discurso, tiene que estar en capacidad de realizar actividades enfocadas al desarrollo de ambientes lúdicos, que permitan goce y disfrute del estudiante a la hora de construir nuevos aprendizajes motrices. Contrario a lo anterior, cuando no se generan ambientes lúdicos desde la clase de Educación Física (en adelante EF), es porque el docente se enfoca en una sola estrategia dinamizadora de la dimensión lúdica, en este caso particular se estaría hablando de un plan de estudios que ve el deporte como un fin y no como un medio para el desarrollo de la motricidad.

En efecto, una clase centrada en ejercicios físicos y actividades de tipo deportivo, en la que se estimula la dimensión lúdica de algunos estudiantes, desconociendo así que otros se aburren de las actividades, de este modo el deseo por aprender o de acercarse al conocimiento se pierde durante las clases, en la cual se pasa a una clase desanimada y monótona, por consecuencia se afecta el proceso de enseñanza-aprendizaje.

El primer responsable de generar ambientes lúdicos es el docente de EF, por lo tanto, la estimulación de la dimensión lúdica recae en él, ya que debe fomentar las buenas prácticas pedagógicas dentro de las clases que aborda, para dar una oportunidad de educar al estudiantado desde algunos componentes lúdicos como, el deporte, el juego motor, las expresiones artísticas y las actividades de evocación o de síntesis.

La etimología de imaginarios proviene del latín "imaginarius", que significa formar una figura mental, por lo tanto, se da desde una mirada desde el individuo, y desde esta perspectiva pluralista, hay que tener en cuenta que, el ser humano también hace sus representaciones en sociedad, por lo que las significaciones imaginarias muestran el modo de ser del por hacer de una determinada organización social. Creando una institución imaginaria de una comunidad específica que se muestra en un tiempo y un espacio histórico-cultural (Miranda Ospino, 2014, p. 17). Hay que aclarar que el Eidos significa forma o figura exterior, por lo que se establece un constructo mental que se desarrolla con imágenes, que también se basa en las actividades diarias que realizan los sujetos dentro de su propia cultura.

Hay que tener en cuenta que desde la perspectiva de Lacan (1953), lo real es inexistente y lo imaginario como percepción de los sujetos que se someten a la realidad, siendo la realidad no como lo real, ya que esta no existe, pero que se nos 
puede presentar una realidad tal cual es, puede que sea algo confuso al momento de diferenciarlo, pero muchas ramas del conocimiento como la psicología y la sociología la diferencia, todo depende del área en donde se va a trabajar, pero Corbin (1993) desde su pensamiento, menciona que todo depende del grado de realidad que se conceda a ese universo imaginado y, por tanto, del poder real reconocido a la imaginación que lo imagina; pero ambas cuestiones están en función de la idea que forman de la representación de lo que se quiere creer.

Los imaginarios han permitido abrir las miradas y crear cultura en el hombre, en este sentido, se convierte en un creador de comunidades y costumbres históricas, pero se convierte en un concepto ambiguo y que no se da desde una racionalidad. "El acceso de este campo se produce siempre de forma indirecta a través de una ambigüedad constitutiva: no lo podemos interpretar según la Lógica de una racionalidad específica, pues no se identifica con el discurso ideológico ni con el deductivo". (Silva, 2006, p. 22).

Según se ha visto, la sociedad es un actor principal de los imaginarios, determina si existe alguna similitud de lo real y lo imaginario o lo imaginario con lo simbólico, y si podrían converger estos significados. Pablo Cazau (1997), en su texto lo real, lo imaginario y lo simbólico, enfoca teóricos como Kant y Jaques Lacan para poder determinar si hay alguna diferencia, o por el contrario hay una similitud, pero que ha entrado en gran debate estos conceptos dentro de la lógica académica, cabe agregar que dentro del marco de la ciencia de la psicología está basado desde un conjunto de saberes conceptuales como lo real, la realidad y lo imaginario.

Castoriadis (1983) menciona que "la institución es una red simbólica, socialmente sancionada, en la que se combinan, en proporción y relación variables, un componente funcional y un componente imaginario" (p. 227). Por otro lado, la institucionalización del imaginario hace parte de la vida, es algo común, no se debate, es por eso que se toma como base para realizar representaciones simbólicas, llegando a desconocer que ellos son, justamente un producto de su propia creación (Murcia, 2012).

Cabe agregar que la educación aborda esta ciencia como referente por sus estudios frente a los procesos de aprendizaje a nivel cognitivo, y es por eso que se debe tomar alguno de los autores ya nombrados para relacionar los imaginarios con la educación, es decir, el constructo mental es realizado por la subjetividad de los individuos y por consiguiente se adapta la idea desde un conjunto de personas para convertirlo en un imaginario simbólico o social, ya como dice Pintos (1995) “(...) Los imaginarios sociales tienen una función primaria que se podría definir como la elaboración y distribución generalizada de instrumentos de percepción de la realidad social construida como realmente existente" (p. 11).

Por otro lado, la dimensión lúdica se ha contemplado en la idea de la sociedad como un sinónimo del juego o actividades recreativas que permiten la diversión de las personas, "La lúdica en este sentido es un concepto poroso, difícil de definir, pero se siente, se vive y se le reconoce en muchas de nuestras prácticas culturales" (Jiménez, 2000 , p.1). Académicamente, la lúdica llega a tener sus raíces desde la ontología y la simbología del ser humano, e inclusive se parte con la acepción de la lúdica históricamente ha generado la identidad de la cultura. "Gran parte de lo que se ha 
dicho sobre la identidad personal también cuenta para identidad cultural. Una cultura o subcultura también muestra una cierta unidad de las partes constitutivas y al mismo tiempo pueden implicar interrupción de a identidad" (Frissen, Lammes, De Lange, De Mul, y Raessens, 2015, p. 30).

En el campo del quehacer pedagógico del docente, debe cumplir con la satisfacción de aprendizaje por medio de la dimensión lúdica, para que los estudiantes tengan un proceso significativo en cada una de las actividades, en este caso son estrategias dinamizadoras. Es por esto que la dimensión lúdica influye en el aprendizaje de los alumnos, por lo que genera un ambiente de aprendizaje por medio del juego, el deporte, las actividades teóricas y las expresiones artísticas.

Siguiendo con el hilo conductor, no es solamente la realización de estas estrategias, ya que es de mayor profundidad, por consiguiente, el docente debe construir un sustento teórico para la realización de esta, en este sentido ayuda en la construcción de un paradigma conceptual para su discurso pedagógico, mitigando de esta forma el activismo o el hacer por hacer.

El funcionamiento que tiene la imaginación simbólica para el docente de EF hace que, durante la planeación de las clases, se realice de forma improvisada, es decir, el concepto de planeación estaría errado para la educación, pero entraría en la perfección en la imaginación simbólica, teniendo en cuenta que este concepto se deriva de dos palabras, el imaginario y el símbolo, el imaginario como lo comenta Solares, (2011, p. 14) es "esencialmente identificado en su concepción con el mito, el arte y el pensamiento religioso de las sociedades tradicionales, constituye, de acuerdo a su pensamiento, el sustrato básico de la vida mental (...)". Lo simbólico, como la forma en la que el hombre interpreta la realidad de una manera distinta, ya que la dota de sentido único. Por todo ello, el objetivo general de esta investigación fue determinar cuál era la imaginación simbólica que tienen los docentes de la ciudad de Villavicencio (Colombia).

\section{MÉTODO}

Para el proyecto de investigación se utilizó el enfoque mixto, por ende, el método expuesto en el estudio fue en el marco de lo descriptivo "Se refiere a un método cuyo objetivo es exponer con el mayor rigor metodológico, información significativa sobre la realidad en estudio con los criterios establecidos por la academia". (Abreu, 2014, p. 198). La relevancia de la aplicación de este método se esgrime por su eficacia en el análisis de la realidad, en este caso, la educación.

\subsection{Muestra}

El estudio realizó una muestra no probabilística de 24 docentes de $E F$, en donde tuvo en cuenta una serie de características que permitieron profundizar la investigación:

- Ser docentes con dedicación exclusiva o parcial a la enseñanza de la Educación Física, Deportes y Recreación de una institución pública de la ciudad de Villavicencio. 
- Ser docente de EF de básica secundaria

- Poseer vinculación de tiempo completo (planta de personal o nombramiento provisional).

De acuerdo con las características, es importante recordar que fue una muestra no probabilística por conveniencia en donde según Iván Espinoza (s.f.): "es la muestra que está disponible en el tiempo o periodo de investigación." (p. 18). El lapso entre los investigados y los investigadores entrarían en relación, ya que permite un adecuado tiempo recíproco en los actores del estudio realizado.

\subsection{Instrumentos}

Para las técnicas e instrumentos de recolección de información se utilizó el diario de campo, la encuesta y la revisión documental, de este modo, se halló una adecuación propia en el estudio para dar solución a la problemática, por lo que la función de las tres son las siguientes:

Diario de campo: la observación y el registro de la práctica cotidiana de los docentes en el lugar de trabajo, a fin de registrar actividades y comportamientos que los mismos no efectúan en forma consciente o son incapaces de registrar; esto permitirá identificar dificultades específicas en la enseñanza por el empleo de modelos de enseñanza, pedagógicos y/o didácticos.

Revisión documental: los documentos facilitan información importante sobre los cuestionamientos realizados en el proceso investigativo. Información importante para el trabajo se puede encontrar en documentos como el PEI, la malla curricular, los planes de clase; en efecto cualquier relato escrito puede ser considerado un documento esencial para la revisión documental, los documentos proporcionan al investigador hechos relacionados con el objeto de la investigación.

Encuesta: se trata de un listado de preguntas que suprime el contacto cara a cara con el investigador. Este tipo de técnica no observacional se da a partir del desarrollo de preguntas escritas que requieren respuestas, la realización de la encuesta fue de forma ad hoc y con preguntas cerradas.

Los instrumentos de recolección de información fueron validados con anticipación por docentes investigadores en el Área de la Educación Física de diversas universidades, para obtener resultados objetivos se tuvo en cuenta la construcción de la misma para poder resolver la pregunta problema en la cual consiste en saber cuál es la imaginación simbólica que tienen los docentes durante su quehacer pedagógico en la ciudad de Villavicencio (Colombia). De igual forma, estos instrumentos permiten la triangulación de la información para poder obtener resultados más específicos, generando a su vez reflexiones en torno al estudio. Para el análisis, se realizó desde la perspectiva del método descriptivo, encontrando especificidades de alguna propiedad, característica y los aspectos más importantes de personas, grupos comunidades 0 cualquier otro fenómeno que es sometido a un análisis (Hernández, Fernández, y Baptista, 2003).

\subsection{Análisis de datos}


Teniendo en cuenta que para el estudio se realizó una triangulación de la información, debido a que hubo instrumentos cuantitativos-cualitativos, la investigación tuvo de referencia a Benavides y Gómez-Restrepo (2005), que mencionan que "la triangulación se refiere al uso de varios métodos (tanto cuantitativos como cualitativos), de fuentes de datos, de teorías, de investigadores o de ambientes en el estudio de un fenómeno." (p. 119). La triangulación respectiva de las técnicas de recolección de información que fueron la revisión documental, la encuesta el cual fue de preguntas cerradas y la observación a través de los diarios de campo. Cabe recordar que "La triangulación de datos puede ser: a) temporal: son datos recogidos en distintas fechas para comprobar si los resultados son constantes; b) espacial: los datos recogidos se hacen en distintos lugares para comprobar coincidencias; c) personal: diferente muestra de sujetos." (Gavira, y Osuna, 2015, p. 74).

El proceso de análisis se enfoco en la contrastación de cada instrumento de recolección de información, además se tuvo en cuenta varios contextos para encontrar coincidencias en los sujetos investigados. Por ende, la veracidad de los instrumentos y la indagación realizada se encuentra basada en el quehacer pedagógico del docente de EF.

\section{RESULTADOS}

\subsection{Encuesta, una mirada desde el imaginario simbólico del docente de EF}

La encuesta se realizó con los 24 docentes integrados en la muestra y se tuvo en cuenta el tema de la imaginación simbólica y el quehacer pedagógico, de este modo se registró algunos datos importantes dentro del interrogante: ¿La malla curricular de su institución cuenta con autores que abordan estrategias lúdicas para el desarrollo de la clase?

Como se muestra en la Tabla 1, el 33\% de los docentes encuestados dijeron que la malla curricular siempre cuenta con autores que sustenten el trabajo realizado durante las clases de EF, mientras que el $21 \%$ dice que casi siempre y el $25 \%$ algunas veces, por último, el $13 \%$ dijo rara vez y el $8 \%$ seleccionó nunca. De acuerdo con este resultado, se hace evidente que el sustento teórico para las Instituciones Educativas es importante para que los docentes no entren en el activismo durante las clases, sino que puedan entrar a un objetivo educativo y académico. Por otro lado, se hizo la pregunta ¿Se basa usted en algún autor a la hora de preparar actividades lúdicas para sus clases? Para saber de este modo si el quehacer pedagógico del docente se basaba en un imaginario simbólico.

Tabla 1. La malla curricular y la imaginación simbólica

\begin{tabular}{ccccc}
\hline Siempre & $\begin{array}{c}\text { Casi } \\
\text { siempre }\end{array}$ & $\begin{array}{c}\text { Algunas } \\
\text { veces }\end{array}$ & Rara vez & Nunca \\
\hline $33 \%$ & $21 \%$ & $25 \%$ & $13 \%$ & $8 \%$ \\
\hline
\end{tabular}


Los resultados de la encuesta (Tabla 2) evidencia que el $21 \%$ afirma que siempre utiliza autores para la preparación de actividades lúdicas, el otro $21 \%$ dice que casi siempre y rara vez, pero hubo un aumento del $33 \%$ en la casilla de algunas veces, por último, el $4 \%$ dice que nunca ha existido un autor dentro de los planes clase.

Tabla 2. Preparación de las actividades lúdicas

\begin{tabular}{ccccc}
\hline Siempre & Casi siempre & $\begin{array}{c}\text { Algunas } \\
\text { veces }\end{array}$ & Rara vez & Nunca \\
\hline $21 \%$ & $21 \%$ & $33 \%$ & $21 \%$ & $4 \%$ \\
\hline
\end{tabular}

En el marco de la práctica docente, se realizó la pregunta que enfoca las actividades educativas, dicho interrogante fue: ¿Utiliza alguna propuesta didáctica para estimular la dimensión lúdica en la clase de EF? De esta manera, se pudo resolver incógnitas investigativas que se encontraban en el proyecto. En concreto, como se presenta en la Tabla 3 , el resultado que se obtuvo fue que en un $33 \%$ siempre, $50 \%$ casi siempre y un $17 \%$ algunas veces, por otra parte, hubo un $0 \%$ que contestaron rara vez y nunca; por lo tanto, dentro de las clases según los docentes de EF existen diversas estrategias didácticas para crear ambientes lúdicos dentro de los aprendizajes de los estudiantes.

Tabla 3. Propuestas didácticas de los docentes

\begin{tabular}{ccccc}
\hline Siempre & Casi siempre & $\begin{array}{c}\text { Algunas } \\
\text { veces }\end{array}$ & Rara vez & Nunca \\
\hline $33 \%$ & $50 \%$ & $17 \%$ & $0 \%$ & $0 \%$ \\
\hline
\end{tabular}

Dentro de la pregunta relacionada con las actividades lúdicas relacionadas con el juego, el $17 \%$ de los docentes de educación eligieron que utilizan siempre actividades lúdicas en clase de EF, mientras que el $62 \%$ respondió que casi siempre (ver Tabla 4)

\begin{tabular}{ccccc}
\hline \multicolumn{5}{c}{ Tabla 4. Las actividades lúdicas desarrolladas en clase } \\
\hline Siempre & Casi siempre & $\begin{array}{c}\text { Algunas } \\
\text { veces }\end{array}$ & Rara vez & Nunca \\
\hline $17 \%$ & $62 \%$ & $17 \%$ & $4 \%$ & $0 \%$ \\
\hline
\end{tabular}

\subsection{Revisión documental desde la labor docente}

Para la técnica de la revisión documental se revisó las mallas curriculares y los planes clase, de este modo se contrastó si la labor del docente EF está basada en una imaginación simbólica

\subsubsection{Planes clase}


Teniendo en cuenta que los planes clase es una estructura en la cual el docente de EF organiza su clase para la enseñanza educativa desde sus saberes, se vuelve importante realizar este tipo de quehaceres pedagógicos. Espontáneamente se induce que no recurre a la improvisación durante de dichas clases, no obstante, dentro del proyecto de investigación denominado "La dimensión lúdica vista desde la imaginación simbólica de los docentes de EF de las instituciones públicas de la ciudad de Villavicencio" se determinó que 17 de los 24 docentes de EF no entregaron los planes clases correspondientes, pero solo cinco (5) realizó la respectiva entrega del documento, dos (2) efectuaron la entrega de documentos que contenían información general acerca de un plan clase, un documento llamado plan anual y otro marco teórico del plan clase.

Se estableció que el $71 \%$ (17) de los docentes no realizan una preparación para efectuar la clase, sino que lo hacen desde un imaginario simbólico que conlleva al docente a realizar activismo y no un mejoramiento en el proceso de enseñanza y aprendizaje. No obstante, se hizo el análisis para determinar si el 29\% (7) restante de los docentes que sí entregaron el plan clase tenía incluida algún referente teórico, el cual refleja el paradigma conceptual que estos tienen frente a los quehaceres pedagógicos.

Se pudo encontrar que tres de los 24 docentes de EF presentan en los documentos un sustento teórico que permite establecer que no están trabajando desde un imaginario simbólico, sino que plantean sus clases con base a la cultura física y el deporte según los siguientes referentes teóricos del área, como lo son José María Cagigal con la cultura intelectual y cultura física, Luis Agosti con, la gimnasia educativa, Kurt Meinel con, la didáctica del movimiento y Dr. Eckert con, Gimnasia, deporte y juego

Por otra parte, las actividades que se encuentran dentro de los planes clase fue principalmente el deporte con un $45 \%$, de este modo se convierte en un sistema automático para las clases de EF, el fútbol, el baloncesto y el voleibol. Son la predominancia de las estrategias dinamizadoras que utilizan en ese porcentaje, en esta ocasión las actividades de síntesis o de evocación con un $27.2 \%$ incluyendo exposiciones, trabajos escritos y consultas durante la clase de EF. Por otra parte, el juego con un $18.1 \%$ en la cual evoca los juegos tradicionales, los lúdicos-recreativos y los juegos predeportivos. Por último, las expresiones artísticas como la danza con un $9 \%$, que aunque se realizan con menor frecuencia esto permite la utilización de diversas estrategias dinamizadoras poco ortodoxas dentro de las clases, para que en este sentido pueda haber unos aprendizajes para los estudiantes.

\subsection{Diario de campo}

En la técnica de observación se llevó a cabo una serie de características para la descripción de la recolección de información, dichas características están compuestas por la estructuración del planeador de clases y la realización de la práctica, es por ello, que la dinámica del docente de EF es un proceso de planificación y actuación dentro del quehacer pedagógico para generar ambientes lúdicos, de este modo se encontraron resultados respectivos dentro del proyecto de investigación. 
Siguiendo con el hilo conductor de la idea, los resultados se dieron con la muestra de 24 docentes y las sesiones de observación que fue de cuatro durante una duración de tres meses, se realizó un análisis profundo frente a los quehaceres educativos y las estrategias dinamizadoras que se dan en el campo de la EF. Es importante recordar que siete (7) docentes efectuaron la entrega de los planes clase, de esta manera, se determinó que dos (2) docentes realizan las clases de acuerdo con lo que planean, mientras que los otros cinco (5) docentes no manejan la temática de acuerdo con la planeación, concluyendo de esta forma que las actividades educativas son en realidad un objeto activista que posiblemente sea de diversión o de goce por parte del docente hacia los estudiantes, ahora bien, los otros 17 docentes no se basan en una planeación de clases para la práctica educativa, sino una acción basada en un imaginario simbólico no teórico.

De acuerdo con la observación realizada se determinó que el juego y el deporte se convierte en una gran influencia para los estudiantes en la estimulación de la dimensión lúdica, manejando en este sentido por parte del deporte innovador en donde el tradicionalismo se ve desplazado y reemplazado por el Ultimate, el Softbol, el Balonmano y Badminton. No obstante, existen algunos momentos en donde el deporte convencional americano-europeo como el fútbol, el baloncesto y el voleibol manejan momentos de felicidad y goce entre los estudiantes, solamente cuando existe una integración y una inclusión por parte del educador hacia el educando se dan dichos instantes. Por otro lado, se refleja que el juego forma parte de un proceso de inclusión y estimulación para generar ambientes lúdicos, por otra parte, las actividades de síntesis y evocación crean aburrimiento dentro del aula de clase, en donde lo primordial es mostrar una información para que los estudiantes puedan utilizar el memorismo y no su imaginación en actividades expuestas mediante el juego, el deporte o las expresiones artísticas.

Finalmente, dentro de las estrategias dinamizadoras en donde los docentes hacen uso desde su imaginación simbólica, se pudo encontrar que el deporte es un elemento primordial para la realización de la clase de EF, utilizando el acondicionamiento físico y los juegos predeportivos para el desarrollo de la misma. Por otra parte, el juego tiende a predominar como segunda estrategia dinamizadora de la que los docentes hacen uso durante las prácticas educativas. Las expresiones artísticas y las actividades de síntesis y evocación son las estrategias dinamizadoras que menos hacen repercusión del quehacer docente de EF, de esta manera se determina que la EF está bajo la mirada de lo deportivizado, pero se refleja algunas opciones no tan ortodoxas como el juego para que los estudiantes tengan otros ambientes educativos y lúdicos.

Son varias las controversias que rodean el proyecto de investigación y que someten al docente de EF como actor principal dentro del ámbito educativo, ya que dentro de su quehacer pedagógico realiza funciones de enseñanza para que los estudiantes sigan en el proceso de aprendizaje, "Si deseamos en el día a día ser docentes, padres de familia, amigos del trabajo, amigos del colegio, entre otros roles con competencias afectivas, es necesario repensarnos y reinventar nuestras actuaciones en medio del proceso de enseñanza y aprendizaje (...)" Padierna, (s.f., p. 14). Existen diferentes estrategias dinamizadoras por lo cual el docente hace uso para 
estimular la dimensión lúdica, ahora bien, no es solamente estimularla para que tengan el mayor grado de felicidad, sino que haya un objetivo de aprendizaje claro dentro del aula o patio de clase.

\section{DISCUSIÓN}

El estudio pretendió determinar cual es la imaginación simbólica que tienen los docentes de la ciudad de Villavicencio (Colombia) en su quehacer pedagógico, por lo que se realizó una triangulación de la información y unos aportes epistemológicos de varias investigaciones que reflejaron una posición similar o contrapuesta para generar reflexiones con base al estudio que se encuentra culminado.

Cuando se realiza la triangulación de la información se obtienen respuestas frente al proyecto de investigación, en esta ocasión, se hizo un análisis riguroso para saber si los docentes laboran desde su imaginación simbólica o por el contrario desde un paradigma conceptual, en este orden de ideas se dieron las siguientes discusiones:

En primera instancia en la encuesta se les preguntó a los docentes si dentro de los planes clase manejan algún autor para desarrollar actividades lúdicas, en donde el $21 \%$ respondió que siempre y otro $21 \%$ dice casi siempre, pero dentro de la revisión documental se demostró que 17 (71\%) docentes no hace una planeación de la clase, ya que no cumplieron con la entrega del mismo documento para efectuar el análisis. En la observación se reflejó la carencia del planeador durante las actividades que estaba desarrollando, en donde, las competencias y los objetivos no se llegan a lograr, teniendo como resultado una gran brecha de la existencia inequívoca en donde los docentes no se enfocan en una estructuración específica para las clases. En esta línea, autores como Ascencio (2016) determinó en una investigación que los objetivos o metas que realice un docente dependerá el resto de los procesos educativos, incluyendo, la estructuración de la clase, la organización, el diseño de la secuencia didáctica, los criterios de evaluación, la bibliografía, y demás. Mencionando esto, el maestro debe tener un discurso hegemónico con base a las lecturas que realizan para poder tener una apropiación de los contenidos que diseña (Enrico, 2011). Razón por la cual, en comparación con los resultados de investigación que enfoco Negles y Negles (2014) en donde el imaginario se encuentra implícito en las prácticas educativas del docente, cuyo aspecto es inconsciente y pragmático, por tal motivo, hay un carencia epistemológica en su quehacer pedagógico, dando hincapié en que el proceso de enseñanza y aprendizaje se fracturaría.

De igual manera, hay similitudes en diversas investigaciones, por ejemplo, Quintero (2011), en sus reflexiones investigativas, menciona que la enseñanza que el maestro efectúan en la escuela, hace que privilegie la dimensión imaginaria de los estudiantes, hacen que evoque la inestabilidad propia de lo simbólico, rechazando toda idea de la realidad del contexto. De acuerdo con lo que se ha comentado, estas fracturas se encuentra arraigadas con los aspectos educativos como el pensamiento crítico, provocando en los estudiantes una realidad imaginaria, puesto que el docente no enfoca su secuencia didáctica en las competencias o en los objetivos de aprendizaje, promoviendo en este sentido el activismo o el hacer por hacer.

En segunda instancia, hay evidencia que, dentro de la observación, los docentes utilizan el deporte para la clase EF, pero existen momentos en donde estimulan la dimensión lúdica. Algunos docentes empiezan a generan actividades de ultimate, softbol o yoga, de igual manera ocurre cuando hay presencia del juego 
durante la práctica educativa. El juego favorece y estimula las cualidades morales de los estudiantes, generando reflexiones en torno a la educación (Minerva, 2002). Siguiendo con los instrumentos de investigación, al contrastarlo con la encuesta, se pudo encontrar que el $55 \%$ de los docentes acuden casi siempre a diferentes estrategias dinamizadoras o didácticas para estimular la dimensión lúdica. En la revisión documental, se refleja varios componentes deportivos, de juego e inclusive de expresiones artísticas, que permite paso a nuevas alternativas en los aprendizajes motrices, y, por consiguiente, llegar a la felicidad y el goce. Las actividades de síntesis no dan respuesta a una clase que dé un estímulo de satisfacción.

Desde la concepción de dimensión lúdica, la satisfacción es importante para el aprendizaje, por lo cual, esta referencia se encuentra argumentada en el estudio de Azofeifa (2006), determinando que una de las motivaciones para encontrar esa satisfacción se encuentra en la imagen corporal, la apariencia, el mantenimiento del peso y el estrés. Por otro lado, Camacho (2003) dice que no importa el tipo de estrategia planteada por el profesor, sino que es la misma actitud del estudiante frente a las actividades, en estos rasgos se pueden incluir los estereotipos, la exclusión y el género, ya que, si existe alguna discriminación en estos ambientes educativos, la estimulación de la dimensión lúdica no se reflejaría en el aula de clase. Para Baños, Ortiz, Baena y Rodríguez (2017) la estructura de la secuencia didáctica debe incluir la motivación hacia el estudiante, para que éste logre los objetivos de aprendizajes planteados por el profesor y alcance a su vez la estimulación de la dimensión lúdica. En el campo de la EF, existen las subjetividades de satisfacción o la relatividad lúdica, en donde cada estudiante tiene su preferencia para sentir felicidad, ya sea en el deporte, el juego, las expresiones artísticas u otras, depende del docente la estructura de los contenidos de aprendizaje, los medios y los mismos objetivos.

Por último, se dio a la respuesta de saber el tipo de estrategia dinamizadora con que los docentes construyen desde su imaginario simbólico, por lo que, dentro del análisis documental y la observación, se concluyó que el deporte es la principal estrategia dinamizadora para los profesores de EF, ya que dentro de la mallas curriculares hay $36 \%$, pero en los planes clase se encuentran con un $45 \%$ y la observación se encontró que hay más preferencia por esta estrategia dinamizadora. Ahora bien, se puede nombrar acerca del tipo de deporte que manejan, ya que se pudo encontrar con diferentes estrategias deportivas en la cual están utilizando, pero se determinó que solo cinco mallas utilizan los deportes convencionales como, el voleibol, el baloncesto, el atletismo, la gimnasia, el futbol de salón o de campo, mientras que las doce mallas restantes hacen uso de otras estrategias deportivas que aunque sean de mínimo uso, permiten crear ambientes lúdicos durante la clase de EF. Dichas estrategias son establecidas en el balonmano, el softbol, el ultimate, el ajedrez, el taekwondo y el bádminton, dicho esto se encuentran en un $70.5 \%$. Al contrastarlo con la encuesta, se observó que los docentes realizan las actividades lúdicas a partir del juego, este porcentaje fue de un $62 \%$ casi siempre, en donde la gran mayoría deciden de esta forma, pero las dos técnicas anteriores reflejan que las actividades que construyen desde su imaginario simbólico están orientadas al deporte. En este sentido, se ha encontrado que la práctica del deporte ayuda al rendimiento académico de los estudiantes, así lo exponen Marín-De la Fuente, Alguacil y Escamilla-Fajardo (2018) en su estudio, donde se comprobó de manera diferencial que los estudiantes que practican algún tipo de deporte tienen mejores resultados académicos, en comparación de los que no practican alguna actividad deportiva. En la misma línea de discusión, conduce los resultados de investigación de Beltrán (2015), que determinó que los estudiantes que se encuentran en proceso de formación para la docencia, prefieren adquirir habilidades en la enseñanza del deporte, por lo que la labor se encuentra ligada por esta estrategia dinamizadora que se encuentra en los espacios 
educativos. Cabe recordar que el objetivo del profesor de EF se basa en la misma formación de sujetos para la transformación del contexto, y no en la formación de deportistas. Se puede dejar al contexto este debate para el análisis y la reflexión, por último, Peñuela (2017) concluye que la didáctica del docente no debe especificarse en una sola estrategia, sino que debe enfocarse en la necesidad de aprendizaje del mismo estudiante.

\section{CONCLUSIONES}

Los siguientes párrafos de conclusión están bajo la perspectiva del muestreo poblacional de los docentes que permitieron la recolección de información y la finalización de la investigación, por lo tanto 24 docentes dan luz para culminar el estudio planteado:

- Se identificó que el deporte es la estrategia dinamizadora en la cual los docentes de EF de Villavicencio (Colombia), construyen su imaginario simbólico sobre la dimensión lúdica, por lo tanto, las clases van orientadas a la dirección de la deportivización, pero que se están empezando a realizar desde un carácter innovador y no convencional.

- Se determinó que los docentes de EF no atienden a los recursos teóricos para la propia argumentación de las actividades planteadas en clase. Aunque existe una minoría que utiliza referencias legales y teóricas se da por hecho que se enfocan desde un imaginario simbólico, a priori o por un supuesto y no en un paradigma conceptual dentro de las Instituciones Educativas y, por consiguiente, se refleja la carencia de discurso pedagógico en los profesores de dicha área.

- Se analizó que los docentes de EF no realizan una planeación frente a las clases, tampoco enfocan los objetivos de aprendizaje para que el proceso de enseñanza no se pierda. Por lo que se culmina en el activismo y la estimulación de la dimensión lúdica por parte de las estrategias dinamizadoras que, en este caso, el deporte y el juego si propenden por ambientes lúdicos cuando la participación total de los estudiantes es activa.

\section{REFERENCIAS}

Abreu, J. L. (2014). El Método de la Investigación. Daena: International Journal of Good Conscience, 9(3), 195-204.

Alonso, M. E. (2009). La Planeación didáctica. (Cuadernos de formación de profesores № 3). Veracruz, México: ENP. Recuperado de http://uiap.dgenp.unam.mx/apoyo pedagogico/proforni/antologias/LA\%20PLAN EACION\%20DIDACTICA.pdf 
Ascencio, C. (2016). Adecuación de la planeación didáctica como herramienta docente en un modelo universitario orientado al aprendizaje. REICE. Revista Iberoamericana sobre Calidad, Eficacia y Cambio en Educación, 14(3), 109130.

Azofeifa, E. G. (2006). Motivos de participación y satisfacción en la actividad física, el ejercicio físico y el deporte. MHSalud, 3(1), 1-16.

Baños, R., Ortiz-Camacho, M. M., Baena-Extremera, A., y Tristán-Rodríguez, J. L. (2017). Satisfacción, motivación y rendimiento académico en estudiantes de Secundaria y Bachillerato: antecedentes, diseño, metodología y propuesta de análisis para un trabajo de investigación. Espiral. Cuadernos del Profesorado, 10(20), 40-50. http://dx.doi.org/10.25115/ecp.v10i20.1011

Beltrán, L. A. (2015). Preferencias deportivas de los estudiantes del programa deportivo formativo en la Universidad Militar sede calle 100 (Trabajo Fin de Máster). Universidad Militar de Nueva Granada, Bogotá (Colombia). Recuperado de https://repository.unimilitar.edu.co/handle/10654/13923

Benavides, M. O., y Gómez-Restrepo, C. (2005). Métodos en investigación cualitativa: triangulación. Revista Colombiana de Psiquiatría, 34(1), 118-124.

Camacho, A. S. (2003). La investigación sobre el pensamiento del alumnado. Una revisión desde la Educación Física. Revista de Educación, 331, 577-613.

Castoriadis, C. (1983). La institución imaginaria de la sociedad (vol.1: Marxismo y teoría revolucionaria). Barcelona: Tusquets.

Cazau, P. (1997). Lo real, lo imaginario, lo simbólico. Revista: El Observador Psicológico No. 24. Julio-agosto. Capital Federal TE.

Corbin, H. (1993). La imaginación creadora en el sufismo de Ibn Arabí. Barcelona: Destino.

Enrico, J. (2011). Un nuevo abordaje del imaginario sarmientino en la configuración de la educación argentina moderna: reflexiones político-conceptuales desde el análisis discursivo (Tesis doctoral). Universidad Nacional de Córdoba, Córdoba (Argentina).

Espinoza, I. (s.f). Tipos de muestreo. Recuperado 10/11/2018 de: http://www.bvs.hn/Honduras/Embarazo/Tipos.de.Muestreo.Marzo.2016.pdf

Frissen, V., Lammes, S., De Lange, M., De Mul, J. y Raessens, J. (2015). Homo ludens 2.0: juego, medios e identidad.

Gavira, S. A., y Osuna, J. B. (2015). La triangulación de datos como estrategia en investigación educativa. Pixel-bit. Revista de Medios y Educación, 47, 73-88. 
Hernández, R., Fernández, C., y Baptista, P. (2003). Metodología de la investigación (3 $3^{a}$ ed.). México: Editorial Mc Graw-Hill.

Jiménez, C. A. (2000). Hacia la construcción del concepto de lúdica. Recuperado el, 12.

Marín-De la Fuente, V., Alguacil, M., y Escamilla-Fajardo, P. (2018). Práctica deportiva extraescolar y su relación con el autoconcepto y el rendimiento académico. eMotion: Revista de Educación, Motricidad e Investigación, 11, 49-61. http://dx.doi.org/10.33776/remo.v0i11.3421

Minerva, C. (2002). El juego: una estrategia importante. Educere, 6(19), 289-296.

Miranda, E. A. (2014). El imaginario social bajo la perspectiva de Cornelius Castoriadis y su proyección en las representaciones culturales de Cartagena de Indias (Tesis doctoral). Universidad de Cartagena, Cartagena de Indias (Colombia).

Murcia, N. (2012). La escuela como imaginario social. Apuntes para una escuela dinámica. Magistro, 6(12), 53-70.

Nagles, E., y Nagles, W. (2014). Imaginarios de los profesores en las prácticas de evaluación (Trabajo Fin de Máster). Universidad de San Buenaventura, Bogotá (Colombia). Recuperado de https://bit.ly/2C3w3IT

Padierna, J. C. (s.f.). Pedagogía y didáctica de la educación física escolar. Recuperado: 4/10/2018 de https://bit.ly/2PD5t1i

Peñuela, C. A. (2017). Didáctica del docente de educación física para la promoción de la actividad física (Tesis doctoral). Universidad del Rosario, Bogotá (Colombia). Recuperado de https://bit.ly/2PCGR8T

Pintos, J. L. (1995). Los imaginarios sociales: la nueva construcción de la realidad social (Vol. 30). Bilbao: Editorial Sal Terrae.

Quintero, C. S. (2011). La enseñanza y la educación del cuerpo infantil: Cuando el lenguaje intercede con" lo vivo". Educación Física y Ciencia, 13, 35-50.

Silva, A. (2006). Imaginarios urbanos. Bogotá: Arango.

Solares, B. (2011). Gilbert Durand, imagen y símbolo o hacia un nuevo espíritu antropológico. Revista Mexicana de Ciencias Políticas y Sociales, 56(211), 1324. 